\title{
Quantum manifestation of Lévy-type flights in a chaotic system
}

\author{
A. Iomin ${ }^{1,2}$ and G. M. Zaslavsky ${ }^{2,3}$ \\ ${ }^{1}$ Department of Physics, Technion, Haifa 32000, Israel \\ ${ }^{2}$ Courant Institute of Mathematical Sciences, \\ New York University, 251 Mercer Str., NY 10012 \\ ${ }^{3}$ Department of Physics, New York University, \\ 2-4 Washington Place, NY, NY 10003
}

\begin{abstract}
Semi-classical dynamics of quantum wave packets spreading is studied for a kicked rotor. Quantum flights are established for a specific, "magic" value of a chaos control parameter when the classical stickiness of trajectories is most effective. By studying of survival probability and distribution of the accelerations we identify the presence of quantum Lévy-type flights.

PACS number(s): 05.45.Mt, 05.60.Gg
\end{abstract}

Lévy or Lévy-type processes occur in different processes related to chaotic dynamics (see reviews in [1 5 ). In Hamiltonian dynamics a phenomenon of stickiness of the chaotic trajectories to island boundaries reveals an intermittent kinetics which can be partly described by Lévy-type processes and fractional kinetic equations [6 [8]. One can find more applications in recent publications 9.10]. Sticking islands can emerge from a tangent bifurcation [11, 12 or from periodic solutions of parabolic type [13,14]. The phenomenon of stickiness to islands reveals in power dependence of the correlation functions, Poincaré recurrences distribution, exit time distribution, etc. [4, 11, 15 [17]. Algebraic dependence of these distributions and moments is not universal; it depends on the models of intermittent dynamics, time intervals, domain of parameters, etc. [6.17], and even using a single characteristic exponent for the algebraic dependence is only a rough approximation to a much more complicated multi-fractal structure of dynamics in chaotic sea in the presence of islands.

Despite the complexity of chaotic dynamics with sticky islands, anomalous diffusion in the form $\left\langle|p|^{\alpha}\right\rangle \sim t^{\beta}$, with momentum $p$ and appropriate exponents $\alpha$ and $\beta$, can often be a good approximation, at least under specified conditions. The case when $\mu=2 \beta / \alpha>1$ is called super-diffusion, while the case $\alpha=2, \beta=1$ corresponds to a normal diffusion. There are different ways to explain the emergence of super-diffusion. One of them, that works for the standard map or the web map, is that a trajectory sticks to an accelerator mode island boundary and travels for a while almost in the same way as a regular (non-chaotic) trajectory of the accelerator mode [8, 11, 15, 17]. As a result, one can observe long flights (Lévy-type flights) in phase space, which corresponds to almost regular pieces of chaotic trajectories. More precise definition of flights refers to the parts of a trajectory with a Lyapunov exponent $\lambda$ fairly small comparing to a typical value of $\lambda$ far from the island boundary layers.

In one way or another, the phenomenon of Lévy-type flights, algebraic correlation decay, stickiness, etc., became objects of intensive studies in quantum physics: Lévy-type processes of scattering and random matrix distributions [18.19] recoils distribution in the laser atoms cooling [20,21]; anomalous transport in the quantum kicked rotor 222 25] and in the Harper model [26]; algebraic decay of the survival probability in different systems [27 29] and in microwave ionization of Rydberg atoms [30,31]; fractal conductance fluctuations [32].

In this paper we consider a quantum kicked rotor (QKR) system, in which the influence of the sticking dynamics on quantum localization process was already demonstrated in [24 for a specific value of the chaos control parameter $K=K^{*}=6.908745 \ldots$, called "magic" number. It has been shown in [15] that the phenomenon of stickiness of a trajectory to the hierarchical islands-around-islands structure is the most efficient at this magic value of $K$. The trajectories from the chaotic sea can travel over long distances in the phase space together with an island if the structure of the boundary islands chain (BIC) is sticky. The stickiness of a trajectory to the hierarchical islandsaround-islands structure is intimately related to magnitude of the parameter $K$. It has been shown in 15 that the effect of non-Gaussian chaotic dynamics (Lévy-type process) becomes more observable when the BIC appears for special magic values of $K$. In particular, for the standard map the value of $K=K^{*}=6.908745$ corresponds to the sequence of islands $3-8-8-8-\ldots$. This exact self-similarity of the islands structure is also reflected in the scaling of the spatio-temporal characteristics of the every island chain generation. A renormalization group was introduced in the form $T^{\prime} \rightarrow \lambda_{T} T, S^{\prime} \rightarrow \lambda_{S} S\left(\lambda_{T}>1, \lambda_{S}<1\right)$, where $S, S^{\prime}$ are the areas and $T, T^{\prime}$ are the periods of the last invariant curves inside of the islands, and these characteristics are related to the islands chain and its next generation. The scaling constants $\lambda_{T}$ and $\lambda_{S}$ determine the transport exponent $\mu=\frac{\left|\ln \lambda_{S}\right|}{\ln \lambda_{T}}$, and this theoretical prediction is in a good agreement with numerics 15. It should be noted that there are no strict conditions on the hierarchy of islands in the case of anomalous transport, and the sequences of values for the islands-around-islands periods can be any numbers permitted by the possible resonances of the system. Each magic value of $K$ generates its own sequence of islands (i.e., it is $3-8-8-8-\ldots$ for $K=6.908745 \ldots$ [15], or $8-8-8-\ldots$ for $K=6.35933 \ldots$ and $5-11-11-11-\ldots$ for $K=6.476939 \ldots$ [17]) and its own scaling constants $\lambda_{S}$ and $\lambda_{T}$. Therefore different hierarchical sequences specify 
different deviations from the Gaussian transport, i.e. normal diffusion, and different characteristic time scales when the anomalous properties of the diffusion can be observed in both classical and quantum cases.

An essential increase in the level occupation amplitudes $\left|f_{n}\right|$ of the order of $10^{5}$ has been found for the magic value $K^{*}$ for strong quantum [24] and semi-classical [25] cases. Anomalous diffusion 23] and the quantum tunneling from the acceleration mode islands [22] for QKR have been studied as well. These effects are implicit indications of Lévy-type flights taking place in the quantum system due to the classical sticking dynamics. Accelerator mode islands move a quantum wave packet in the energy levels space of the unperturbed system faster than in the case of global chaos, since the Lyapunov exponents in the vicinity of these islands are considerably smaller than in the chaotic sea. Hence a trajectory sticks to this hierarchical area for long time. The question of direct observation of these flights is still open, and we consider this problem here.

We study numerically the anomalous transport for the Hamiltonian of the QKR

$$
H=\frac{p^{2}}{2}+K \cos q \delta_{T}(t)
$$

where $p=-i \tilde{h} \frac{\partial}{\partial q}$ is the angular momentum and $q \in[0,2 \pi]$ is the angle, while $\tilde{h}$ is the dimensionless Planck constant, $K$ is the control parameter, and $\delta_{T}(t)=\sum_{n} \delta(t-n)$ is a periodic train of $\delta$ - kicks with the period $T=1$. The quantum map

$$
\Psi(t+T)=\hat{U}(T) \Psi(t)
$$

is produced by the following evolution operator over the period $T$ :

$$
\hat{U}=\exp \left\{-i \tilde{h} \hat{n}^{2} / 2\right\} \exp \{-i(K / \tilde{h}) \cos q\} .
$$

Below we study the evolution of the complex amplitudes for the expansion of the wave function $\Psi$ in terms of the unperturbed system basis $e^{i n q}$,

$$
\Psi(t)=\Psi(q, t)=\sum_{n=-\infty}^{\infty} f_{n}(t) e^{i n q}
$$

Here $\left|f_{n}(t)\right|^{2}$ is the probability of level occupation at time $t$ which determines the spreading of the energy growth over the unperturbed spectrum. It enables us to study the evolution of survival or staying probability in an energy region with momentum range $\Delta p=\tilde{h} \Delta N$, which is not necessarily compact, and determined without constraints on the boundaries:

$$
P(t)=\sum_{n \in \Delta N}\left|f_{n}(t)\right|^{2}
$$

For iteration of the map (2) we use the standard technique of the fast Fourier transform with up to $2^{18}$ angular momentum eigenstates. We study also the time evolution of the second moment $\sigma_{2}=\left\langle p^{2}\right\rangle=\tilde{h}^{2} \sum_{n}\left|f_{n}\right|^{2} n^{2}$ as a function of $K$ and the initial conditions. The initial conditions for the amplitudes of level occupation are $f_{n}(t=0)=$ $\delta_{n, n^{\prime}}$ where $n^{\prime}=0$ corresponds to the initial population inside acceleration islands, while $n^{\prime}= \pm 31$ correspond to the initial population in chaotic sea with $p \approx \pm \frac{3 \pi}{8}$ for $\tilde{h}=0.0396$. The values of $\tilde{h}$ are irrational numbers or rational approximations of irrational numbers such that quantum resonances with $\sigma_{2} \sim t^{2}$ are avoided.

We started the study of the wave packet spreading for $f_{n}(t=0)=\delta_{n, 0}$. When the chaos parameter $K>1$ approaches the magic number $K^{*}=6.908745 \ldots$ the anomalous super-diffusion takes place in the energy space. In this case, sticking dynamics inside of the hierarchical islands around islands structure spreads the initial wave packet very fast. This leads to the anomalously diffusive growth of the second moment $\sigma_{2}(t) \sim t^{\beta}$, where $\beta \approx 1.57 \pm 0.27$ in the time interval $t \in[0,300]$. Then the transport exponent changes to diffusive one $\beta \approx 0.98 \pm 0.04$ determined in the time interval $t \in[400,1000]$ (see Fig. 1). The diffusion coefficient $D \sim 10^{3}$ is anomalously large and differs essentially from normal diffusion coefficient $K^{2} / 4 \ll D$.

The super-diffusion for $K=K^{*}$ is also determined by an increase of $\left|f_{n}\right|$ by more than $10^{10}$ times in comparison with $\left|f_{n}\right|$ corresponding to usual diffusion with $K=9.69$ (see Fig. 2). This differences can be explained by quantum flights, which reflect Lévy-type flights in the classical limit. To illuminate these flights we compute survival probability in periodic energy level regions taken in periodic classical cells of the size $2 \pi \times 2 \pi$. To that end $\frac{\tilde{h}}{2 \pi}=\frac{1}{157+g m}$ is determined by a rational approximation of the golden mean value: $g m=55 / 34$, and one obtains for the dimensionless Planck constant $\tilde{h}=2 \pi \cdot R / Q=2 \pi \cdot 34 / 5393$. Therefore $\tilde{h}$ is commensurate with energy level spacing with the period of 
5393 levels, which corresponds to the 34 cells of the size $2 \pi$ on a cylinder. One takes 40 levels in every periodic cell and studies the change of $P(t)$ in (5) with time, where

$$
\Delta N=\bigcup_{l=-a}^{a}[l \cdot Q+\operatorname{sgn}(l), l \cdot Q+\operatorname{sgn}(l) \cdot 40]
$$

and $a$ is an integer part of the ratio $N / 2 Q$. The result of the numerical calculation is shown in Fig. 3. Every passage of the wave packet through the periodic cell contributes to the increase of survival probability $P(t)$. This causes the spikes of $P(t)$. The period between any two spikes is equal to the number of periodic cells: $\Delta t=R=34$. This means that the part of the wave packet which sticks to the BIC passes through one cell per one iteration, i.e. it moves ballistically. Amplitudes of the spikes decay exponentially with time by the following expression $\sim \exp (-0.005 t)$. It relates to the quantum tunneling through cantori [22,33,34]. This behavior of quantum tunneling from the accelerator mode to the chaotic sea is in a good agreement with the result obtained in 22, 35.

The spikes disappear if the initial conditions are chosen inside of the chaotic sea with $n^{\prime}= \pm 31$ for $f_{n}(t=0)=\delta_{n . n^{\prime}}$. Nevertheless, super-diffusion due to the sticking to BIC dynamics takes place for $K=K^{*}$ with $\sigma_{2} \sim t^{\beta}$, where $\beta \approx 1.39 \pm 0.16$. Also, in comparison with $K=9.69$, an increase of order of magnitude in the level occupation amplitudes $\left|f_{n}\right|$ is obtained. It is the same order as shown in Fig. 2. These differences unambiguously indicate the presence of flights. To pinpoint these quantum flights, we calculate a set of the survival probabilities $P_{k}(t)$ constructed over a set of inclosed compact regions of the energy levels $\Delta N(k)=N_{0} k$, where $N_{0}=3^{6}$ and $k=1 \div 10$ are integer numbers. Let us denote the time intervals which are necessary for the wave packet to reach the boundaries of $\Delta N(k)$ as $t_{k}$. In our numerical calculations $t_{k}$ is the number of iterations. We found a linear growth of the times $t_{k}$ which is proportional to the increase of size of the regions $\Delta N(k)$, such that $t_{k} \sim \Delta N(k)$ (see Fig. 4(c)). It means that the part of the wave packet which reaches the boundary of $\Delta N(k)$ at the moment $t_{k}$ moves like in an acceleration mode: $\Delta p \sim \tilde{h} \Delta N \sim \Delta t$. As far as the diffusive spreading of the wave packet for $K=9.69$ goes, one can see in Fig. 4(a) that in this case $t_{k}$ is not proportional to $\Delta N(k)$. Another important characteristic of the wave packet evolution is the decay rate of the survival probability. Starting from $t_{k}$ the survival probability $P_{k}\left(t \geq t_{k}\right)$ in (5) decays in correspondence with the rate of the spreading of the wave packet. The decay rate depends on $K$. In the chaotic case when $K=9.69$ only the tail of the wave packet moves ballistically. It corresponds to the transitions between any two levels $n_{1}$ and $n_{2}$ such that $\left|n_{1}-n_{2}\right| \sim K / \tilde{h}$. In this case the matrix elements of the evolution operator (3), $U_{n_{1}, n_{2}}=e^{-i n_{1}^{2} \tilde{h} / 2} J_{n_{1}-n_{2}}(K / \tilde{h}) \sim \sqrt{\tilde{h} / K}$, where $J_{m}(z)$ is the Bessel function of the first kind, are small and uncorrelated random quantities. Their total contribution to the transition is summing up to an exponentially small value, which is reflected by the exponentially small values of $\left|f_{n}\right|$ in Fig. $2(\mathrm{a})$. Therefore, $P_{k}(t)$ decay gradually and slowly with time as it is shown in $4(\mathrm{a}, \mathrm{c})$. For $K=K^{*}$ the values of $P_{k}(t)$ fall down abruptly e.i. much faster then in the chaotic case, which is illustrated in Fig. 4(b,d). It means that there is a part of the wave packet that moves ballistically together with the tail. Obviously that this part determines the decay of $P_{k}(t)$. Therefore, in case of mixed phase space the hierarchical sticking islands structure leads to ballistic flights. Fast change of $P_{k}(t)$ in Figs. 4(b,d) relates to these quantum flights, while the slow changes of $P_{k}(t)$ in Figs. $4(\mathrm{a}, \mathrm{c})$ relate to normal diffusion.

It should be noted that the classical nature of the phase space reflects also in pure quantum characteristics of the evolution operator $\hat{U}$ (3). Let us show that the matrix elements corresponding to flights, such that $\left|n_{1}-n_{2}\right| \sim K / \tilde{h}$ are much stronger correlated for $K \approx 2 \pi$ than for $K=9.69$. We consider the following correlation function:

$$
\rho(\eta)=\sum_{n}\left\langle\left\langle U_{m, n} U_{n, m+\eta}^{*}\right\rangle\right\rangle_{m},
$$

where $\langle\langle\ldots\rangle\rangle_{m} \equiv \frac{1}{L} \sum_{m=1}^{L} \ldots$ means averaging over $m$ in some energy levels region, such that $1 \ll L \ll 1 / \tilde{h}$. By using the explicit form of the matrix elements $U_{m, n}$, one obtains for the correlation function

$$
\rho(\eta)=\frac{1}{L} e^{-i \tilde{h} \eta^{2} / 2} J_{\eta}(2 K / \tilde{h}) \frac{e^{i \tilde{h} \eta L}-1}{1-e^{i \tilde{h} \eta}}
$$

Let us take $\eta=[k / \tilde{h}]=K / \tilde{h}-\{K / \tilde{h}\}$, where $[Z]$ is the integer part, while $\{Z\}$ is the fractional part of $Z$. Therefore $\tilde{h} \eta=K-\tilde{h}\{K / \tilde{h}\}=K-\varepsilon$, and the condition $L \varepsilon \ll 1$ is also fulfilled. Hence, it is easy to see from (8), that if $K=2 \pi+\varepsilon($ or $K=2 \pi n+\varepsilon)$, then $\rho(\eta) \equiv \rho_{a c c}([K / \tilde{h}]) \sim J_{[K / \tilde{h}]}(2[K / \tilde{h}])$ is the correlation function with presence the acceleration mode motion in the classical counterpart. It is also easy to see for the global chaos case (including $K=9.69)$, that the correlation function satisfies $\rho(\eta) \equiv \rho_{\text {diff }}\left([K / \tilde{h}]=\frac{1}{L} \rho_{a c c}([K / \tilde{h}]) \ll \rho_{\text {acc }}([K / \tilde{h}])\right.$. It means that for the diffusive case without any stability islands the matrix elements are fairly less correlated than in the case of 
anomalous diffusion, in which the acceleration mode islands (with $K=2 \pi n+\varepsilon$ ) play crucial role for both classical and quantum transport and quantum localization of anomalous diffusion [25]. It should be noted that for $K=2 \pi$ the motion is diffusive both for classical and quantum case. Only for $2 \pi<K<7$ the anomalous diffusion has been found. Similarly to our result, the anomalous diffusion for QKR has been found in [22] and [23] for $K=2 \pi \cdot 1.03$ and $K=6.9115$ correspondingly. This range of $K$ relates to bifurcate emerging and existing of the acceleration mode islands. That fact that the quantum correlation functions (7) reflects the sticking dynamics in the vicinity of the hierarchical structure of these islands relates to the fundamental correspondence principle by which the semi-classical wave packet evolves alone an individual trajectory during the quantum correspondence time $\tau_{h}$ 36, 24, 25. The $\tilde{h}$ scaling for this time is algebraic, $\tau_{h} \sim \tilde{h}^{\frac{-1}{1+\beta}} \sqrt[37]{3]}$ for the acceleration mode dynamics. This result corresponds to general consideration of quantum dynamics with mixed phase space 28,38]

To complete this analysis of quantum flights, we consider the acceleration statistics. These values are defined as the mean square momentum changes at every iteration,

$$
\delta p(t) \equiv\left\langle\left(p_{t+1}-p_{t}\right)^{2}\right\rangle / K^{2}=\left\langle\sin ^{2} q_{t}\right\rangle,
$$

where $\langle\ldots\rangle$ means the quantum average. It can be expressed by the level occupation amplitudes in the following form:

$$
\delta p(t)=\sum_{n}\left[\frac{1}{2}\left|f_{n}(t)\right|^{2}-\frac{1}{4} f_{n}^{*} f_{n-2}-\frac{1}{4} f_{n}^{*} f_{n+2}\right] .
$$

One should note that the dynamical consideration is restricted in time because of the fast spreading of the wave packet. This leads to the fast overfill of energy levels region with the fixed size of $N=2^{18}$ levels. Therefore, the maximal number of iterations is restricted and equals to 1000 for $\tilde{h}=0.0399$. Dynamical calculations of $f_{n}(t)$ are carried out for a set of initial conditions, arbitrary chosen inside the chaotic sea to obtain an appropriate statistics.

Let us consider probability distribution function $W_{K}(\delta p)$ of accelerations $\delta p$. For the white noise $W_{K}(\delta p)$ is Gaussian, while for a process which corresponds to the fractional kinetics, one expects $W_{K}(\delta p) \sim 1 /(\delta p)^{l}$, where $|\delta p| \rightarrow \infty$. The exponent $l=\log _{10}\left(W_{K}(\delta p) / \log _{10}(\delta p)\right.$ determines to what kind of statistics $W_{K}(\delta p)$ belongs. When $l$ is small enough, we associate the distribution $W_{K}(\delta p)$ with the Lévy-type probability distribution function, and vice versa, if $l$ is large, $W_{K}(\delta p)$ is associated with the Gaussian one, since one cannot distinguish between large $l$ and exponentially small values of $W_{K}(\delta p)$. The distribution of accelerations $\Delta p=\delta p-\overline{\delta p}$ for the set of 36 different initial conditions with $31<\left|n_{0}\right|<70$, arbitrary chosen inside the chaotic region is plotted in Fig. 5 . Here $\overline{\delta p}$ is the mean value averaged over $t$.

The probability distribution functions are asymmetric and we compare only the right tails of the chaotic dynamics with $K=9.69$ and the sticky case with $K=K^{*}$. The distribution function for chaotic, non-sticky case has a short right tail with a large slope of $l \approx 9.5$. This magnitude of $l$ is large enough, and one concludes that the semi-classical diffusion gives rise to Gaussian distribution for the accelerations. The distribution function for $K=K^{*}$ differs essentially from the Gaussian distribution. As it is shown in Fig. 5, it is narrower with a longer tail and the slope is of the order of $l \approx 4.3$. These differences enable us to conclude that $W_{K=K^{*}}(\Delta p)$ is the Lévy-type distribution and relates to the quantum flights which are due to the effect of stickiness of the semi-classical wave packet to the classical hierarchical islands-around-islands structure.

This research is supported by U.S. Department of Navy Grants No N00014-96-10055, No N00014-97-1-0426 and by U.S. Department of Energy Grant No DE-FG02-92-ER54184. A.I. was also supported by the Israel Science Foundation.

[1] E. W. Montroll and M. F. Shlesinger, in "Studies in Statistical Mechanics", edited by J. Leibowitz and E. Montroll (North-Holland, Amsterdam, 1984), vol. 11, p. 1.

[2] J. P. Bouchand and A. Georges, Phys. Rep. 195, 127 (1990).

[3] "Lévy Flights and Related Topics in Physics", edited by M. Shlesinger, G. M. Zaslavsky, and U. Frisch (Springer-Verlag, Heidelberg, 1995).

[4] M. F. Shlesinger, G. M. Zaslavsky, and J. Klafter, Nature 363, 31 (1993).

[5] T. Geisel, in [3], p. 153.

[6] V. Afanasiev, R. Z. Sagdeev, and G. M. Zaslavsky, Chaos 1, 143 (1991).

[7] G. M. Zaslavsky, Physica D 76, 110 (1994); Chaos 4, 25 (1994).

[8] G. M. Zaslavsky and B. A. Niyazov, Phys. Rep. 283, 73 (1997). 
[9] P. Leboeuf, Physica D 116, 8 (1998); S. S. Abdullaev and K. H. Spatschek, Phys. Rev. E 60, R6287(1999);

[10] G. Zimbardo, P. Veltri, and P. Pommois, Phys. Rev. E 61, 1940 (2000).

[11] J. D. Meiss, et al., Physica D 6, 375 (1983).

[12] J. D. Meiss, Phys. Rev. A 34, 2375 (1986); Rev. Mod. Phys. 64, 795 (1992).

[13] V. Melnikov, in "Chaos, Transport, and Plasma Physics", edited by S. Benkadda, F. Doveil, and Y. Elskens (World Scientific, Singapore, 1994), p. 126.

[14] V. Rom-Kedar and G. M. Zaslavsky, Chaos 9, 6973 (1999).

[15] G. M. Zaslavsky, M. Edelman, and B. A. Niyazov, Chaos 7, 159 (1997).

[16] B. V. Chirikov and D. L. Shepeliansky,Physica D 13, 395 (1984); B. V. Chirikov, Chaos, Solitons, Fractals 1, 79 (1991).

[17] G. M. Zaslavsky and M. Edelman, Chaos 10, 135 (2000); S. Benkadda, et al., Phys. Rev. E 55, 4909 (1997); 59, 3761 (1999).

[18] D. Kusnezov, Phys. Rev. Lett. 72, 1990 (1994); D. Kusnezov and C. H. Lewenkopf, Phys. Rev. E 53, 2283 (1999).

[19] D. Kusnezov, A. Bulgak, and G. D. Dang, Phys. Rev. Lett. 82, 1136 (1999).

[20] F. Bardou, J. P. Bouchaud, O. Emile, A. Aspect, and C. Cohen-Tannoudji, Phys. Rev. Lett. 72, 203 (1994); B. Saubaméa, M. Leduc, and C. Cohen-Tannoudji, Phys. Rev. Lett. 83, 3796 (1999).

[21] S. Schaufler, W. P. Schleich, and V. P. Yakovlev, Phys. Rev. Lett. 83, 3162 (1999).

[22] J. D. Hanson, E. Ott, and T. M. Antonsen,Jr., Phys. Rev. A 29, 819 (1984).

[23] R. Roncaglia, L. Bonci, B. J. West, and P. Grigolini, Phys. Rev. E 51, 5524 (1995); L. Bonci, P. Grigolini, A. Laux, and R. Roncaglia, Phys. Rev. A 54, 112 (1996).

[24] B. Sundaram and G. M. Zaslavsky, Phys. Rev. E 59, 7231 (1999).

[25] A. Iomin and G. M. Zaslavsky, Chaos 10, 147 (2000).

[26] A. Iomin and G. M. Zaslavsky, Phys. Rev. E 60, 7580 (1999).

[27] Y.-C. Lai, R. Blümel, E. Ott, and C. Grebogi, Phys. Rev. Let. 68, 3491 (1992).

[28] G. Casati, G. Maspero, and D. L. Shepelyansky, Phys. Rev. Let. 82, 524 (2000).

[29] G. Casati, I. Guarneri, and G. Maspero, Phys. Rev. Let. 84, 63 (2000).

[30] A. Buchleiner, et al., Phys. Rev. Lett. 75, 3818 (1995).

[31] G. Beneti, G. Casati, G. Maspero, and D. L. Shepelyansky, cond-mat/9911200.

[32] R. Ketzmerick, Phys. Rev B 54, 10841 (1996).

[33] T. Geisel, G. Radons, and J. Rubner, Phys. Rev. Let. 57, 2883 (1986).

[34] Our result is in a good agreement with 22], where the quantum tunneling from the acceleration mode island has been studied for variety of $\tilde{h}$ and $K=2 \pi(1.03) \approx 6.4717$. Using [22] one obtains for $\tilde{h}=\frac{2 \pi \cdot 34}{5393}$ that the decay rate due to quantum tunneling is $\exp (-5.3) \approx 0.005$.

[35] We thank E. Ott for drawing our attention to this result.

[36] G. P. Berman and G. M. Zaslavsky, Physica A 91, 450 (1978).

[37] A. Iomin and G. M. Zaslavsky, unpublished.

[38] R. Ketzmerick, L. Hufnagel, F. Steinbach, and M. Weiss, Phys. Rev. Lett. 85, 1214 (2000); L. Hufnagel, R. Ketzmerick, and M. Weiss, cond-mat/0009010. 


\section{FIGURE CAPTIONS}

Fig. 1 Time evolution of the second moment $\sigma_{2}$ for initial population inside the acceleration mode island: $n^{\prime}=0$, and $\tilde{h}=\frac{2 \pi \cdot 34}{5393}$, where (a) $K=9.69$, and (b) $K=K^{*}$.

Fig. 2 Level occupation amplitudes $\left|f_{n}\right|$ vs $n$ for $K=9.69$ (a) and $K=K^{*}$ (b) after 1000 iterations. The slop of the linear plot for the energy region $(6 \div 12) \times 10^{4}$ is $1.4 \cdot 10^{-5}$.

Fig. 3 Semi-log plot of the survival probability $P(t)$ vs $t$ for periodic cells with the period $64 \pi$. The plots are: (a) for $K=9.69$, and (b) for $K=K^{*}$.

Fig. 4 A set of survival probabilities $P_{k}(t)$ vs $t$ for $K=9.69$ (a,c) and $K=K^{*}$ (b,d) for variety of energy regions $\Delta N(k)=3^{6} \cdot k$ and $k=1 \div 10$ and $\tilde{h}=2 \pi /(157+g m)$ Plots (c) and (d) zoom in of (a) and (b) respectively for $k=3$.

Fig. 5 (upper plots) Probability distribution functions $W_{K}(\Delta p)$ for 120 boxes and the centered by $\Delta p=\delta p-\overline{\delta p}$, where $\overline{\delta p}$ is the mean value with $K=9.69$ for (a); and $K=K^{*}$ for (b). (bottom plots) Slops for the right tails of the probability distribution functions, where (a) corresponds to $K=9.69$ and the slop is $9.51 \pm 0.02$ for $\Delta p \in[0.0111,0.0146]$; (b) corresponds to $K=K^{*}$ and the slop is $4.31 \pm 0.06$ for $\Delta p \in[0.0109,0.0156]$. 


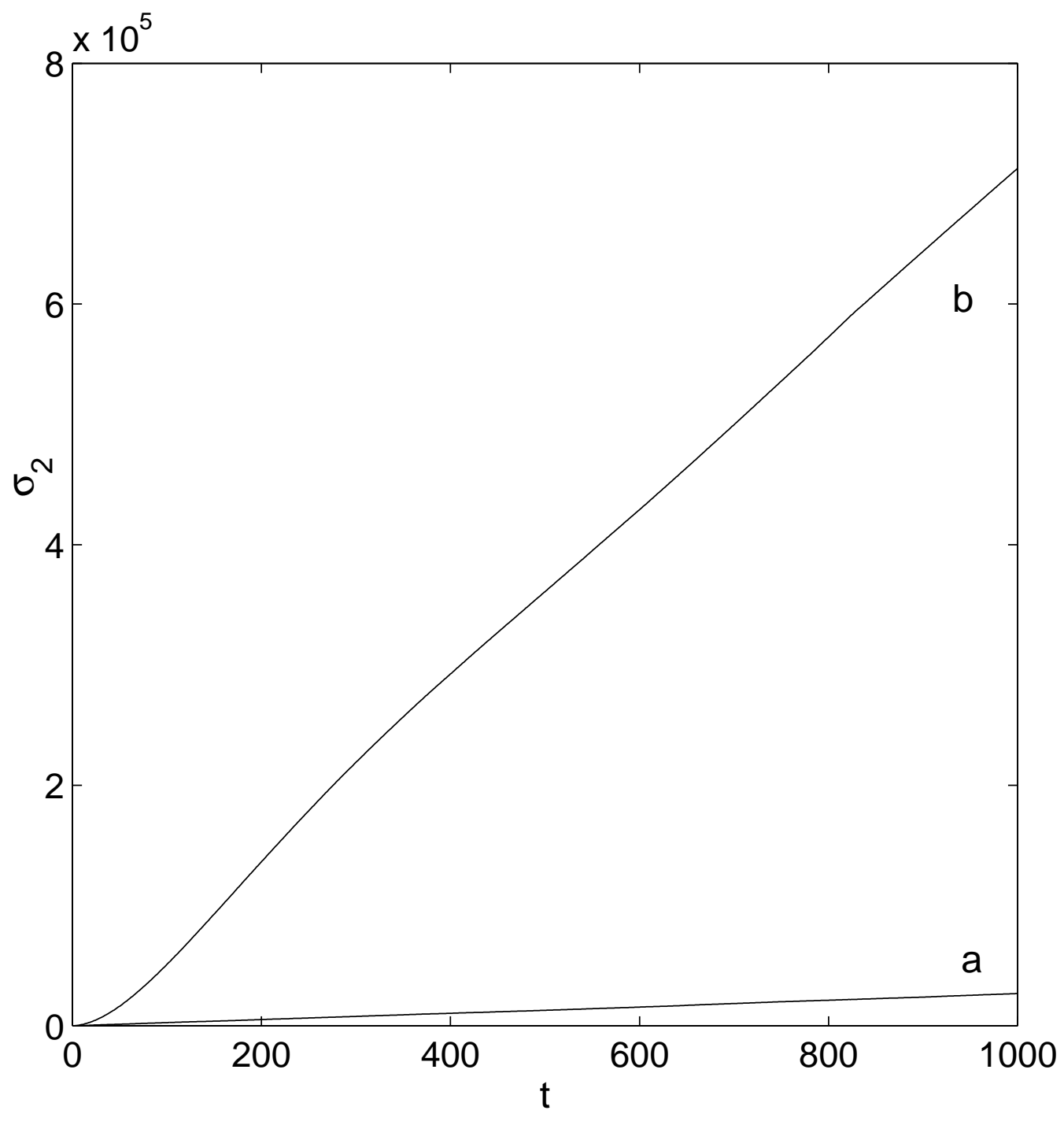




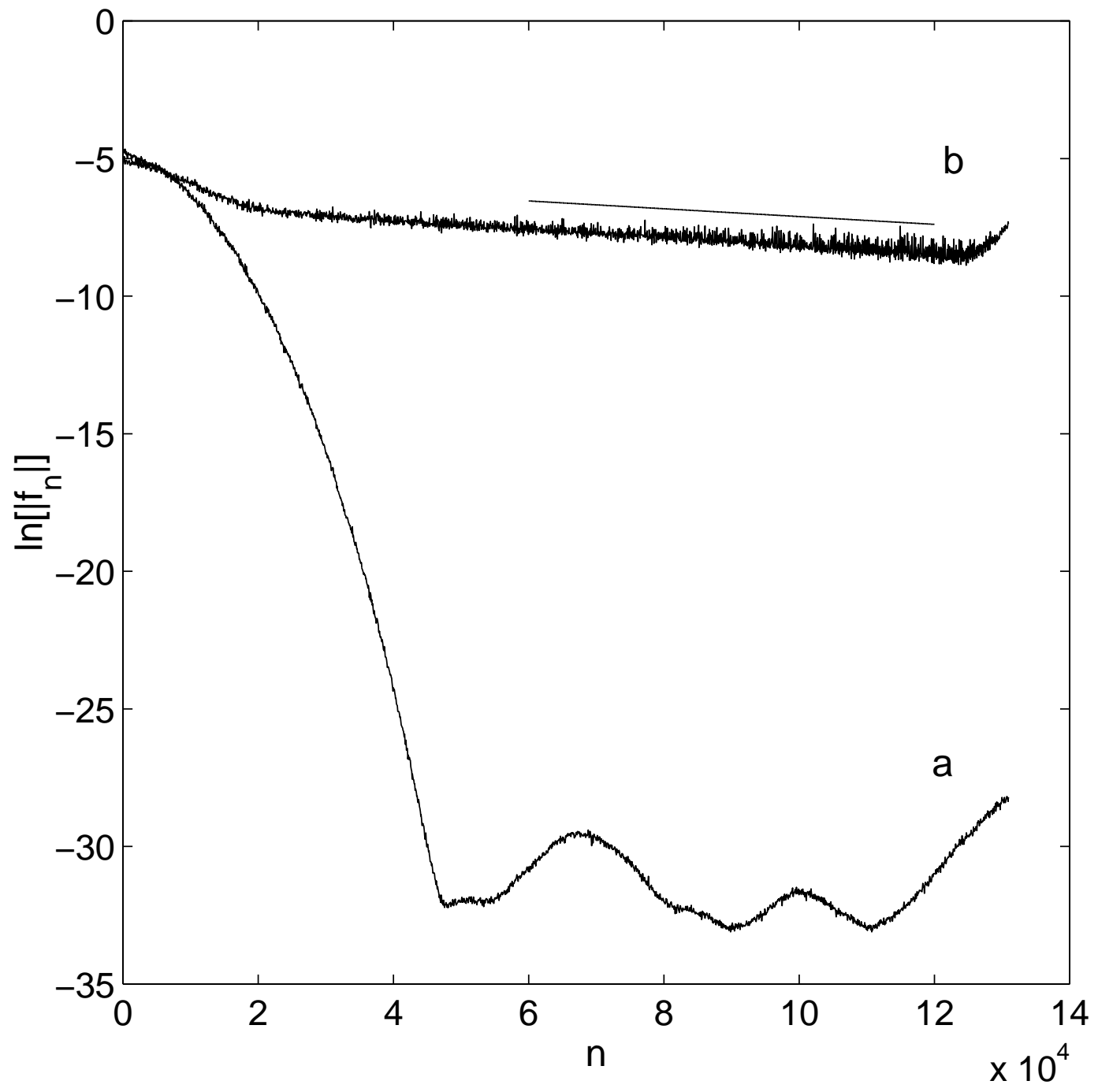



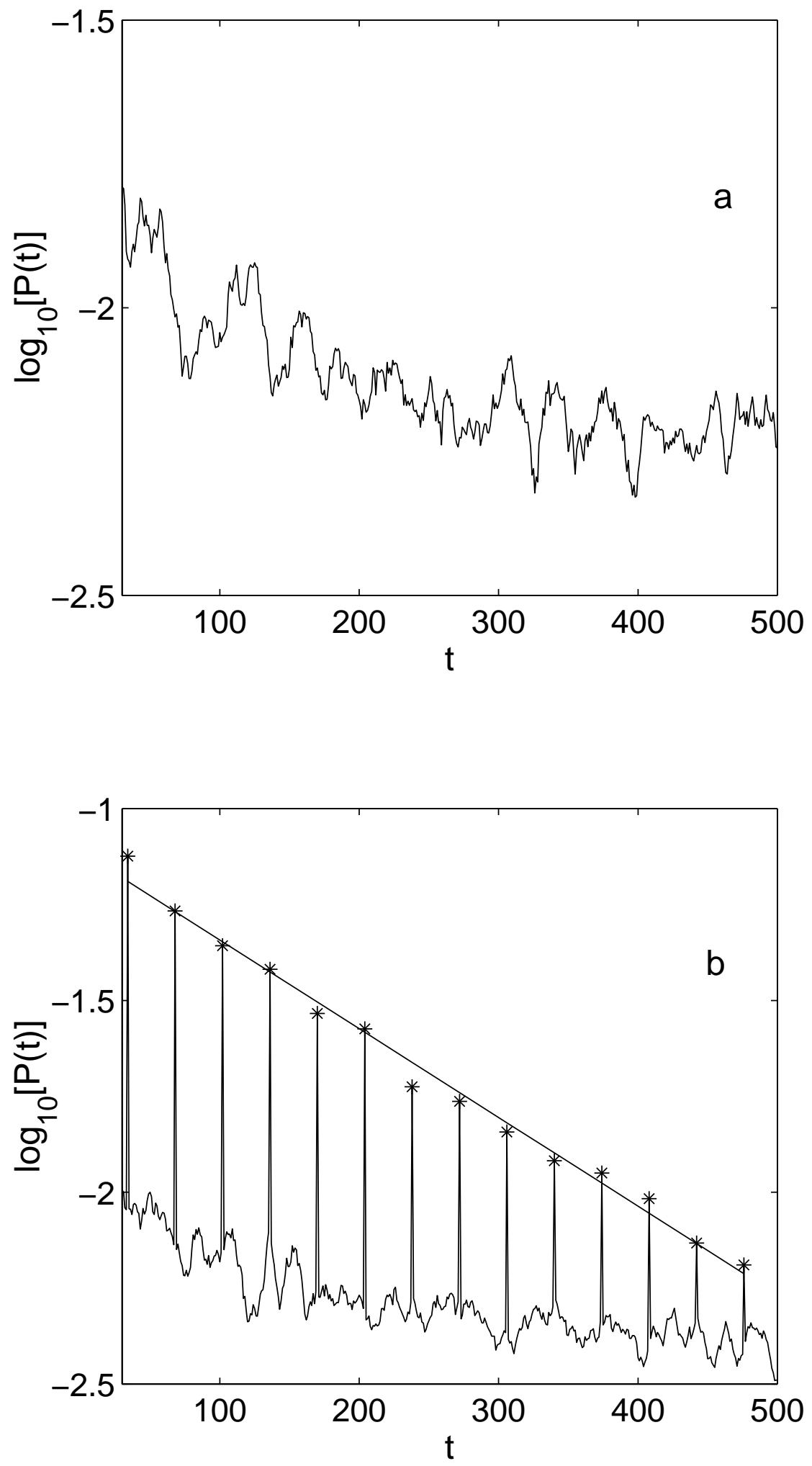

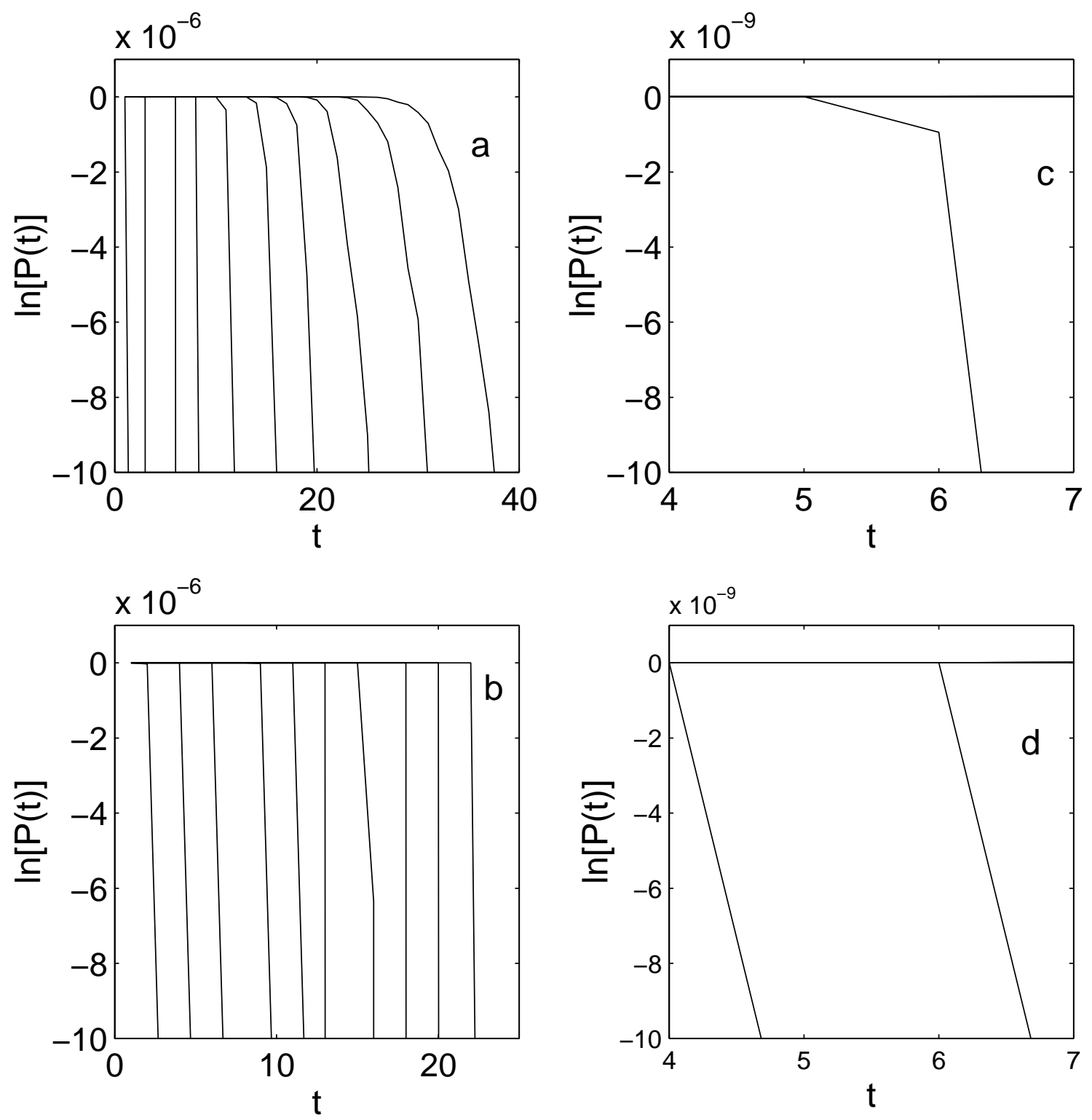

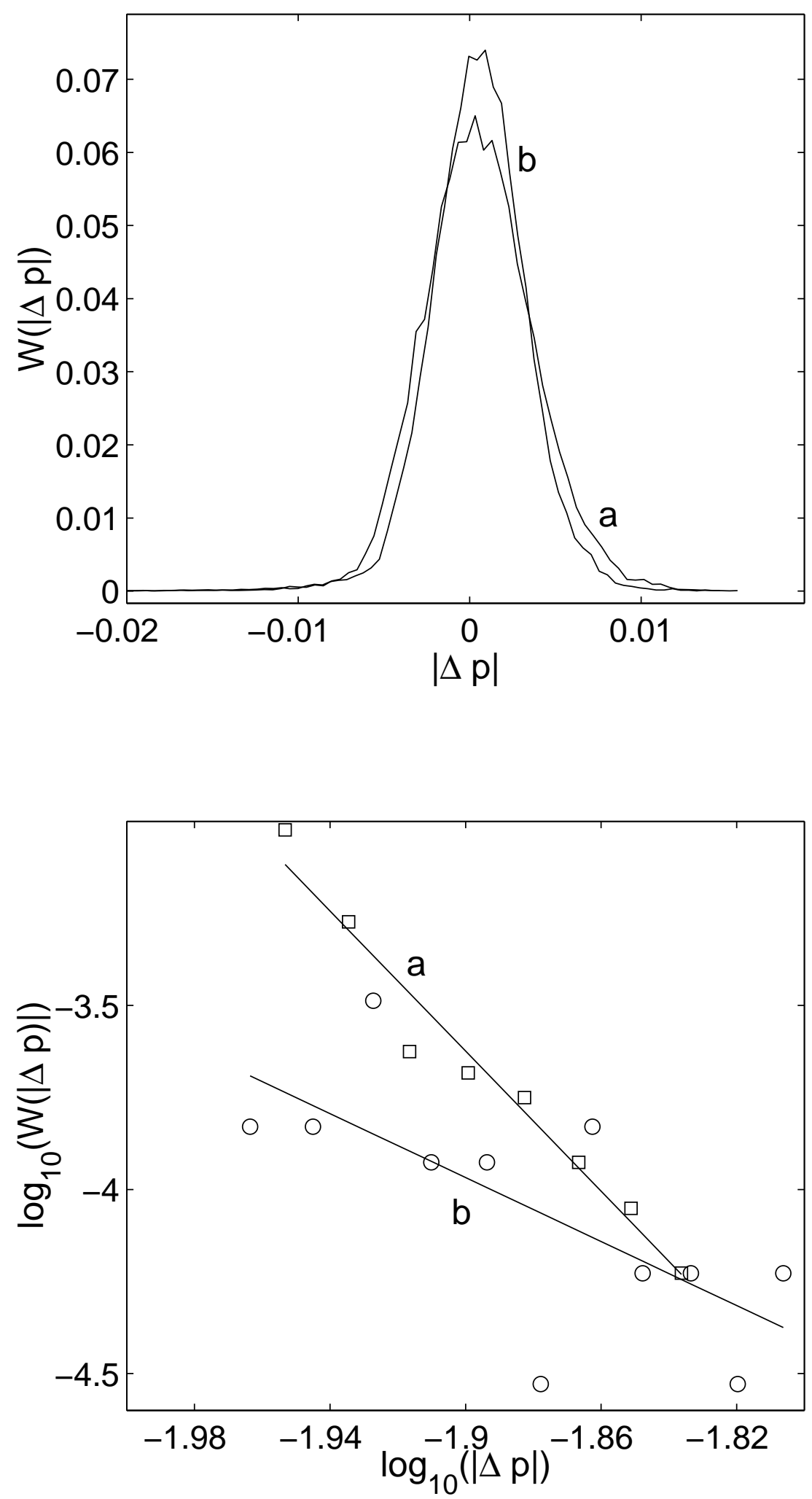